\title{
Correction to: Activation of an Endogenous Opsin 3 Light Receptor Mediates Photo-Relaxation of Pre-Contracting Late Gestation Human Uterine Smooth Muscle Ex Vivo
}

\author{
Peter D. Yim ${ }^{1}$ - Shunsuke Hyuga ${ }^{1} \cdot$ Amy D. Wu ${ }^{1} \cdot$ William Dan $^{1} \cdot$ Joy Y. Vink ${ }^{2} \cdot$ George Gallos $^{1}$ \\ Published online: 15 July 2020 \\ (C) Society for Reproductive Investigation 2020
}

Correction to: Reproductive Sciences https://doi.org/10.1007/s43032-020-00180-z

This article was updated to correct Joy Y. Vink's name in the author listing.

The online version of the original article can be found at https://oi.org/ 10.1007/s43032-020-00180-Z

$\triangle$ Peter D. Yim

pdy1@ columbia.edu

1 Department of Anesthesiology, Vagelos College of Physicians and Surgeons, Columbia University, 622 168th Street, P\&S Box 46, New York, NY, USA

2 Department of Obstetrics and Gynecology, Vagelos College of Physicians and Surgeons, Columbia University, New York, NY, USA 